\title{
1 Dynamic and stationary brain connectivity during movie watching as revealed by functional MRI
}

2 Xin $\mathrm{Di}^{1 *}$, Zhiguo Zhang ${ }^{2,3}$, Ting Xu ${ }^{4}$, Bharat B. Biswal ${ }^{1 *}$

3

4 1. Department of Biomedical Engineering, New Jersey Institute of Technology, Newark, NJ, 07102, USA

5 2. School of Biomedical Engineering, Health Science Center, Shenzhen University, Shenzhen, China

6 3. Guangdong Provincial Key Laboratory of Biomedical Measurements and Ultrasound Imaging,

7 Shenzhen, China

8 4. Center for the Developing Brain, Child Mind Institute, New York, NY 10022, USA

9

$10 *$ Corresponding author:

11 Xin Di, Ph.D.

12604 Fenster Hall, University Height

13 Newark, NJ, 07102, USA

14 xin.di@njit.edu

15

16 Bharat B. Biswal, Ph.D.

17607 Fenster Hall, University Height

18 Newark, NJ, 07102, USA

19 bbiswal@yahoo.com

20

21

22

23 Running title: Dynamic and stationary connectivity 


\section{Abstract}

Spatially remote brain regions show synchronized activity as typically revealed by correlated functional MRI (fMRI) signals. An emerging line of research has focused on the temporal fluctuations of connectivity, however, its relationships with stationary connectivity have not been clearly illustrated. We examined dynamic and stationary connectivity when the participants watched four different movie clips. We calculated point-by-point multiplication between two regional time series to estimate the timeresolved dynamic connectivity, and estimated the inter-individual consistency of the dynamic connectivity time series. Widespread consistent dynamic connectivity was observed for each movie clip, which also showed differences between the clips. For example, a cartoon movie clip, The Present, showed more consistent of dynamic connectivity with the posterior cingulate cortex and supramarginal gyrus, while a court drama clip, A Few Good Men, showed more consistent of dynamic connectivity with the auditory cortex and temporoparietal junction, which might suggest the involvement of specific brain processing for different movie contents. In contrast, the stationary connectivity as measured by the correlations between regional time series was highly similar among the movie clips, and showed fewer statistically significant differences. The patterns of consistent dynamic connectivity could be used to classify different movie clips with higher accuracy than the stationary connectivity and regional activity. These results support the functional significance of dynamic connectivity in reflecting functional brain changes, which could provide more functionally related information than stationary connectivity.

Keywords: dynamic connectivity; movie connectome; movie watching; naturalistic stimuli; stationary connectivity 


\section{Introduction}

47 The human brain exhibits a highly synchronized structure of activity as revealed by functional MRI

48 (fMRI) in resting-state (Biswal et al., 1995, 2010), during task performance (Cole et al., 2014; Di et al.,

49 2020; Krienen et al., 2014), and during watching naturalistic stimuli such as movies (O'Connor et al.,

50 2017; Vanderwal et al., 2019). Functional connectivity, as measured by the correlations of observed

51 blood-oxygen-level-dependent signals (Biswal et al., 1995; Friston, 1994), have been widely used to

52 examine the organization of large-scale brain networks (Margulies et al., 2016; Salvador et al., 2005; Yeo

53 et al., 2011) and to parcellate small brain structures such as the thalamus and striatum (Di Martino et al.,

54 2008; Tian et al., 2020; Yuan et al., 2016). However, the spatial distribution of functional connectivity is

55 highly similar across different tasks and movie watching conditions (Cole et al., 2014; Di et al., 2020;

56 Krienen et al., 2014; Vanderwal et al., 2019). To localize functionally meaningful connections, it is

57 therefore critical to examine the time-varying fluctuations of connectivity (Allen et al., 2014; Di and

Biswal, 2020; Hutchison et al., 2013), as well as the changes of functional connectivity between different

task conditions (Di and Biswal, 2019; Fornito et al., 2012; Friston et al., 1997).

61 window approach (Allen et al., 2014; Hutchison et al., 2013; Lurie et al., 2020). It has been shown that

62 the variability of dynamic connectivity fluctuations is lower between regions from the same functional

63 networks and higher between regions from different networks (Fu et al., 2017), resulting in an overall

64 negative correlation with the stationary functional connectivity (Thompson and Fransson, 2015; Zhang et

65 al., 2018). However, because of the unconstrained nature of the resting-state, it is difficult to ensure that

66 the obtained dynamic connectivity estimates are functionally meaningful or simply resulting from noise

67 (Lindquist et al., 2014). Until recently, dynamic connectivity is also studied when the participants were

68 given complex stimuli, such as watching movie clips (Di and Biswal, 2020). The advantage of using a

69 movie stimulus is that the time course of dynamic connectivity can be compared across participants. If

70 there are high inter-individual similarity (Hasson et al., 2004; Nastase et al., 2019), then it may imply that 
71 the observed dynamic connectivity is functionally meaningful and is relevant to the processing of the

72 video stimuli.

In our previous study, we have demonstrated the inter-individual consistency of dynamic

74 connectivity when different participants watched the same animated movie Partly Cloudy (Di and Biswal, 2020). By using a seed-based analysis, we identified highly consistent dynamic connectivity between the supramarginal gyrus and posterior cingulate gyrus, two regions that are critical in the processes of empathy and theory of mind (Richardson et al., 2018). Moreover, among a set of regions of interest, the dynamic connectivity pattern was largely dissociated with the stationary functional connectivity that was measured by the correlations of the time series from the entire run. For example, the stationary functional connectivity between the supramarginal gyrus and posterior cingulate gyrus was close to zero, while the windowed dynamic connectivity showed highly consistent fluctuations. To date, only handful of studies have examined dynamic connectivity during movie watching (Cooper et al., 2021; Di and Biswal, 2020; Freitas et al., 2020; Simony and Chang, 2020). It is still largely unknown how the spatial pattern is modulated by different movie contents, and how dynamic connectivity is spatially distributed.

The central goal of this study is to compare dynamic connectivity and stationary connectivity in the context of movie watching. In addition to the previously analyzed Partly Cloudy dataset (Richardson et al., 2018), we also analyzed the Healthy Brain Network Serial Scanning Initiative (HBN-SSI) dataset (O’Connor et al., 2017), where same participants watched three different movie clips. The video clips were derived from different types of movies, ranging from a science fiction cartoon comedy, a science fiction action film, to a court drama. It is reasonable to expect that different brain systems are involved in the process of the different movie clips. However, Vanderwal and colleagues have examined the stationary connectivity of the three movies, and showed very similar spatial patterns among them (Vanderwal et al., 2019). We speculate that dynamic connectivity might be more sensitive to reflect the changes in brain functions among the movie clips.

Further, we systematically examine the relationships between dynamic and stationary connectivity in terms of their spatial distributions and context modulations. The economic theory of brain 
97

98

99

111 We analyzed two publicly available fMRI datasets when participants watched different movie clips, the

112 Partly Cloudy dataset (Richardson et al., 2018) and the HBN-SSI dataset (O'Connor et al., 2017). For the

113 Partly Cloudy dataset, we analyzed the adults' data where they watched the animated movie "Partly

114 Cloudy". And for the HBN-SSI dataset, we analyzed the data when the same participants watched three 115 different movie clips from different types of movies.

\section{$116 \quad$ 2.1.1. Partly Cloudy dataset}

117 The Partly Cloudy data were obtained through openneuro (https://openneuro.org/; accession \#:

118 ds000228). Consistent with our previous study, we only included the adult participants $(\mathrm{n}=33)$ (Di and

119 Biswal, 2020). After dropping data due to large head motion (see below) and poor brain coverage, the

120 effective sample included 17 females and 12 males. The mean and standard deviation of age were 24.6

121 years and 5.3, respectively (age range: 18 to 39 years). The original study was approved by the 
122 Committee on the Use of Humans as Experimental Subjects (COUHES) at the Massachusetts Institute of 123 Technology. (Pixar, 2009). MRI images were acquired on a 3-Tesla Siemens Tim Trio scanner with the standard

126 Siemens 32-channel head coil. Blood-oxygen-level dependent (BOLD) sensitive fMRI images were

127 collected with a gradient-echo EPI sequence in 32 interleaved near-axial slices (EPI factor: 64; TR: 2 s,

128 TE: $30 \mathrm{~ms}$, and flip angle: $90^{\circ}$ ). The participants were recruited for different studies with slightly

129 different voxel sizes and slice gaps. Three participants had $3.13 \mathrm{~mm}$ isotropic voxels with no gap, and 26

130 participants had $3.13 \mathrm{~mm}$ isotropic voxels with a $10 \%$ gap. All the functional images were resampled to 3

$131 \mathrm{~mm}$ isotropic voxel size during preprocessing. 168 functional images were acquired, with four dummy

132 scans before the real scans to allow for steady-state magnetization. T1-weighted structural images were

133 collected in 176 interleaved sagittal slices with $1 \mathrm{~mm}$ isotropic voxels (GRAPPA parallel imaging,

134 acceleration factor of 3; FOV: $256 \mathrm{~mm}$ ). More information can be found in Richardson et a. (2018).

\subsubsection{HBN-SSI dataset}

The HBN-SSI dataset was obtained through the project website

137 (http://fcon_1000.projects.nitrc.org/indi/hbn_ssi/). Thirteen participants were recruited in the study.

138 After removing data of four participants due to excessive head motion in any of the movie-watching

139 sessions, data from four females and five males were included in the current analysis. All the participants

140 are right-handed. The age range was from 23 to 37 years old (Mean $=29.4 ; S D=5.5)$.

We selected the movie watching scans of three movie clips, Wall-E (Walt Disney Productions,

142 2008), The Matrix (Warner Bros., 1999), and A Few Good Men (Columbia Pictures, 1992), from the 12

143 repeated scanning sessions. Each movie clip was 10 minutes long and was watched by the same

144 participant four times in separate sessions. The order of the movie watching was counterbalanced across

145 sessions. The fMRI data were scanned using an EPI sequence with the following parameters, TR: 1,450

$146 \mathrm{~ms}$, TE: $40 \mathrm{~ms}$, flip angle: $55^{\circ}$, and voxel size: 2.46 x $2.46 \mathrm{~s} 2.5 \mathrm{~mm}^{3}$ without any gap. Four hundred and

147 twenty images were scanned for each run. However, for one participant, there were only 410 images for 
several sessions. We, therefore, used the first 410 images for the current analysis for all the subjects and sessions.

Lastly, the MPRAGE image from the first sequential scanning session of each participant was also used to assist preprocessing of the fMRI and DWI data. The scanning parameters include TR, 2,730 ms; TE $1.64 \mathrm{~ms}$; flip angle, $7^{\circ}$; voxel size $1 \times 1 \times 1 \mathrm{~mm}^{3}$ with no gap. More information about the study design and MRI acquisitions can be found in O'Connor et al., (2017).

\subsection{FMRI data preprocessing}

The fMRI data preprocessing was performed by using SPM12 (SPM, RRID: SCR_007037) under

MATLAB environment (https://www.mathworks.com/). The two datasets were preprocessed using very similar pipelines. Specifically, the anatomical image of each participant was first segmented into gray matter, white matter, cerebrospinal fluid, and other tissue types, and normalized into standard Montreal Neurological Institute (MNI) space. The functional images of each session and subject were aligned to the first image of their specific session and were coregistered to the skull stripped anatomical image of the subject. The deformation field maps obtained from the segmentation step were used to normalize all the functional images into MNI space. The fMRI images from the Partly Cloudy dataset were resampled to 3 x $3 \times 3 \mathrm{~mm}^{3}$ voxel size; and the images from the HBN-SSI dataset were resampled to $2.5 \times 2.5 \times 2.5 \mathrm{~mm}^{3}$, which were chosen according to their respective original voxel sizes. All the functional images were then spatially smoothed using an $8 \mathrm{~mm}$ Gaussian Kernel. Lastly, we defined a generalized linear model (GLM) for each session and subject by using 24 head motion variables (Friston et al., 1996) and a constant term as regressors, with implicit high-pass filtering at 1/128 Hz. After model estimation, the residual images were saved for further analysis.

We calculated framewise displacement for translation and rotation for each session and participant (Di and Biswal, 2015). For the Partly dataset, we used strict criteria of maximum framewise displacement of $1.5 \mathrm{~mm}$ or $1.5^{\circ}$ to discard data with large head movements. Two participants' data were discarded accordingly. For the HBN-SSI dataset, a participant's data were discarded if any of the sessions exceeded the criteria. We adopted a slightly liberal criterion of maximum framewise displacement 
greater than $2.5 \mathrm{~mm}$ or $2.5^{\circ}$ or mean framewise displacement greater than $0.2 \mathrm{~mm}$ or $0.2^{\circ}$. Four

175 participants' data were removed accordingly.

176

177

178

179

180

181

182

183

184

185

186

187

188

189

190

\subsection{Independent component analysis}

Because the main goal of the current study is to study connectivity across brain regions, we adopted spatial independent component analysis (ICA) to define connectivity nodes. We extracted 20 and 80 ICs to represent different spatial scales of brain networks. We first analyzed the local activity and connectivity with 20 -IC solutions to identify statistically significant local effects. We then calculated connectivity using the 80-IC solutions to examine their spatial distributions. For spatial ICA, the number of ICs that could be extracted depends on the number of time points for each participant/session.

Theoretically, $t$ - 1 components can be extracted where $t$ represents the total number of time points. We chose 80, which is roughly half of the time points for the Partly Cloudy dataset. Group ICA of fMRI Toolbox v3.0b (Group ICA of fMRI Toolbox, RRID: SCR_001953) was used for ICA (Calhoun et al., 2001). The ICA was performed for the Partly Cloudy and HBN-SSI datasets separately. After extraction, we manually selected the ICs that were related to functional networks and discarded the noise-like ICs. For the Partly Cloudy dataset, 16 and 65 ICs were considered functionally meaningful ICs for the 20-IC and 80-IC solutions, respectively. And for the HBN-SSI dataset, 16 and 54 ICs were kept. After ICA, the time series of each IC for each subject and session were back reconstructed by using the group ICA algorithm. The time series were used for further activity and connectivity analyses.

\subsection{Inter-individual consistency of regional activity}

For both regional activity and dynamic connectivity, we estimated the inter-individual consistency across participants and sessions. Conventionally, the intersubject correlation was used to study the interindividual consistency (Chen et al., 2016; Hasson et al., 2004; Nastase et al., 2019). In our recent study, we have shown that the principal component analysis (PCA) can be used to estimate the inter-individual consistency, which is quantitively similar to intersubject correlation (Di and Biswal, 2021). Specifically, for a given region we have a $t$ (\# of time points) by $n$ (\# of participants) matrix $X . X$ is a $168 \times 29$ matrix 
for the Partly Cloudy dataset and a 410 x 36 matrix ( $36=9$ participants $\times 4$ sessions) for each of the three

201

202

movie clips from the HBN-SSI dataset. We performed PCA on the matrix $X$ and obtained the percent variance explained by the first PC as a measure of intersubject consistency.

For the HBN-SSI dataset, there were four sessions for each participant and each movie. Ideally, the multi-session and multi-participant design can be used to differentiate the consistent and idiosyncratic responses. We have explored this issue and found that the within-participant consistency was mainly driven by the overall across-participant consistency, but not participant-specific idiosyncratic responses (see supplementary materials). Moreover, the idiosyncratic responses are not the focus of the current study. Therefore, in the current analysis, we treated session and participant as separate data and calculated inter-individual consistency across all the sessions and participants.

\subsection{Dynamic connectivity}

The sliding-window approach is the most commonly used method to estimate dynamic connectivity (Allen et al., 2014; Di and Biswal, 2020; Fu et al., 2014). A recent development is to utilize point-bypoint multiplications of two time series to approximate their dynamic connectivity, a.k.a. edge-centric time series (Faskowitz et al., 2020). The development comes from the intuition that the commonly used measure of functional connectivity, i.e., Pearson's correlation coefficient, is the summation of the pointby-point multiplications of two $\mathrm{z}$ transformed variables divided by the sample size minus 1 . Therefore, if we keep the original point-by-point multiplication time series, it can reflect estimates of dynamic connectivity at every time point. In Figure 1, we show the averaged time series of two networks, i.e., the posterior cingulate network and supramarginal network from the Partly Cloudy dataset. The averaged sliding-window and point-by-point multiplication time series were also shown. Strong negative multiplication values can be seen when the two original time series have strong anti-phase cofluctuations. Indeed, the peaks in the posterior cingulate network and supramarginal network represent the theory-of-mind and pain empathy events, respectively, as indicated by the original paper (Richardson et al., 2018). The point-by-point multiplication indicates strong negative connectivity during these events. In contrast, the sliding window correlation can only reflect a smoothed trend of such interactions. 
The point-by-point multiplication approach can avoid overly smoothing the time series data as done by

227 the sliding-window approach, therefore providing better interpretability of the results. On the other hand,

228 the multiplication term may be noisier and more prone to physiological noises and head motion artifacts.

229 But this may be less problematic for movie watching data, where we can estimate the consistent effects

230 cross individuals. We have performed statistical analyses on the Partly Cloudy dataset, and confirmed

that the point-by-point multiplication approach had better statistical sensitivity (see Supplementary connectivity.

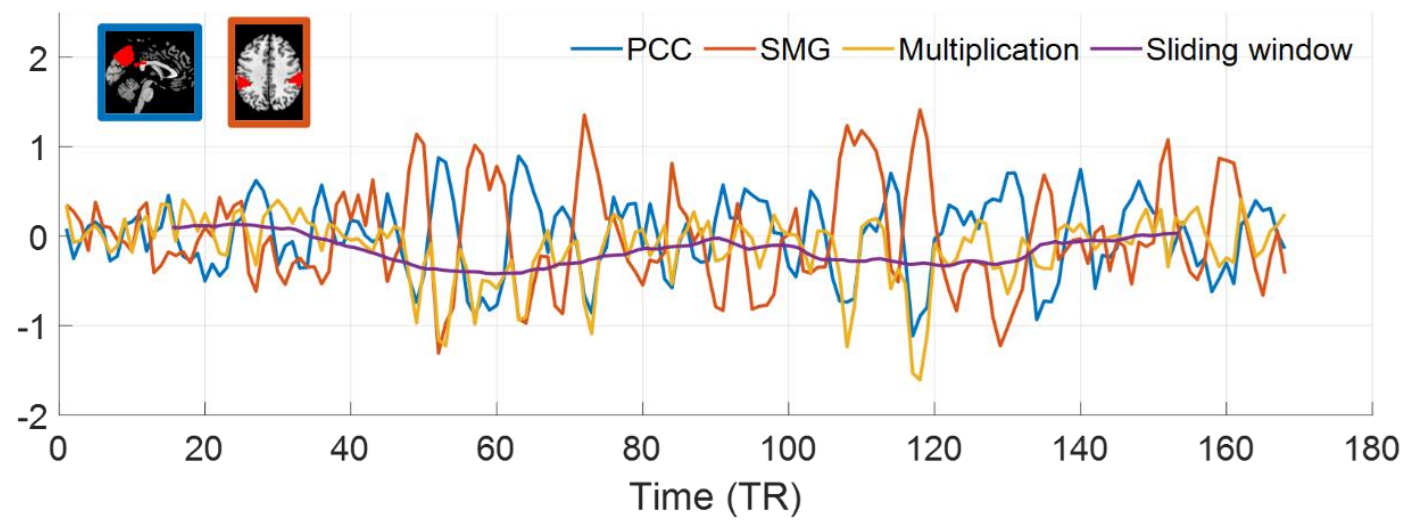

Figure 1 Averaged time series of regional activity in the posterior cingulate (PCC) network and

We calculated the point-by-point multiplication between each pair of networks (ICs). The time

241 series from each network (IC) were first $\mathrm{z}$ transformed, and then point-by-point multiplied. PCA was

242 then performed on the multiplication time series across participants. To determine the statistical

243 significance of the variance explained by the first PC, we performed circular time-shift randomization to

244 determine the null distribution (Di and Biswal, 2021; Kauppi et al., 2010). The time series from the two

245 network ICs from all the participants were circular-shifted with random delays. Point-by-point 
multiplications were then calculated for each participant, and PCA was performed. The randomization

247 was performed 10,000 times for each pair of networks from the 16 networks. The real values were

248 compared with the null distribution to perform statistical inferences. This resulted in a $16 \times 16$ matrix.

249 False discovery rate (FDR) correction was used to correct for multiple comparisons (120: 16 x 15 / 2).

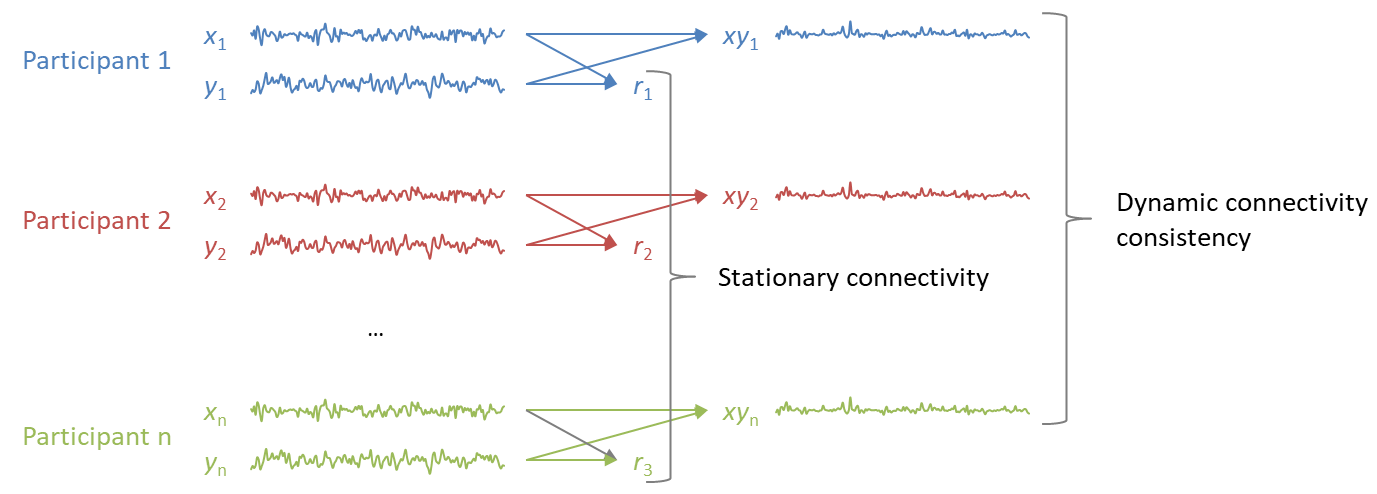

Figure 2 Illustration of the calculation of the consistency of dynamic connectivity and stationary connectivity.

For the HBN-SSI dataset, we also compared the differences in variance explained by the first PC among the three movies. For a pair of networks ICs, the multiplication between two IC time series were first calculated, forming a 410 (time point) x 36 (participant/session) matrix for each movie. The matrices

257 from the three movie clips were concatenated to a 410 x 108 matrix, and permutation was performed 258 along the individual/session dimension to define three permutated matrices. The differences in variance explained by the first PC between each movie clip and the other two clips were calculated and compared with the permutated distributions of 10,000 times. FDR correction at $\mathrm{p}<0.05$ was used to correct 261 multiple comparisons of all three movie clips.

The randomization-based statistics were performed for all the analyses in the 20-IC solutions.

263 For the 80-IC solution, the goal of the analyses was not to identify specific statistically significant

264 connections. Rather, we examined the spatial distributions of the dynamic connectivity, and their 265 relations to stationary connectivity. 


\subsection{Relations to stationary connectivity}

267 Next, we examined how the spatial distribution of consistent dynamic connectivity is associated with

268 stationary connectivity. Here we focused on a finer spatial scale of 80 -IC solutions. To assess the

269 stationary connectivity, we calculated Fisher's z transformed Pearson's correlations across the included

270 networks (ICs). The matrices were averaged across individuals, and transformed back to $\mathrm{r}$ quantities.

271 First, we examined whether the dynamic and stationary connectivity has similar spatial distributions. For

272 both matrices, the upper triangular part was converted to vectors, which were in turn correlated with each

273 other between different movie clips. We adopted Spearman's correlation coefficients to avoid violations

274 of Gaussian distributions of the matrix data.

275 Next, we calculated connectivity gradients (Margulies et al., 2016; Vos de Wael et al., 2020)

276 based on the stationary connectivity patterns in the HBN-SSI dataset. By calculating gradients, the brain

277 networks (ICs) can be placed into a 2-D space based on their relative stationary connectivity strengths.

278 The 2-D gradients reflect large-scale brain organizations between unimodal networks and higher-order

279 transmodal areas (e.g. the default mode network) and between visual and sensorimotor regions (Margulies

280 et al., 2016). We can next display dynamic connectivity in the 2-D space to illustrate whether the

281 dynamic connectivity takes place between proximal or distal regions in the 2-D space. Specifically, we

282 first calculated the gradients for each movie clip based on the group stationary connectivity matrices

283 using the BrainSpace toolbox (Vos de Wael et al., 2020). The gradients were then aligned across the

284 movie clips with Procrustes alignment. The default diffusion-embedding and row-wise threshold (top

$28510 \%$ percentile) were used. After model fitting, the first two gradients were obtained. The network ICs

286 were mapped into the 2D gradient space. Lastly, the dynamic connectivity from the three movie clips

287 was plotted on the 2-D layout.

\section{$288 \quad$ 2.7. Movie clips classification}

289 In addition to univariate analysis, we also explored whether the dynamic connectivity, stationary

290 connectivity, and regional activity can reliably reflect an individual's movie-watching condition (Finn et

291 al., 2015). The analysis was performed based on the 54 network ICs from the 80-IC solution. For each 
participant of the HBN-SSI dataset, we calculated a connectivity or activity measure for each movie clip, and also the corresponding connectivity or activity measure for the remaining 8 participants. We compared three types of measures. First, we calculated the inter-individual consistency of point-by-point multiplications. The individual's consistency was calculated across the four sessions of the same movie clips. The consistency of the remaining participants was calculated across 32 participants/sessions. The

297 lower diagonal of the matrices was converted into a $1,431(54 \times 53 / 2)$ by 1 vector to perform the

298 classification analysis. Second, we calculated mean stationary connectivity for the individual (averaged 299 across 4 sessions) and the remaining participants (averaged across 32 sessions). Third, we calculated the inter-individual consistency of the regional activity. Similarly, individual measures were calculated across the four sessions, and the remaining participants' measures were calculated for the 32 procedure to randomly shuffle the predicted movie label 10,000 times.

\section{3. Results}

\section{3.1. Dynamic and stationary connectivity in 20-IC solution}

315 We first examined the dynamic connectivity by calculating point-by-point multiplications between each

316 pair of 16 networks (IC) on the Partly Cloudy dataset (Figure 3a). We found widespread inter-individual

317 consistent effects at $\mathrm{p}<0.05$ of FDR correction. We also applied the sliding window approach, which 
only yielded statistically significant effects on six pairs of networks (supplementary materials). This

suggests that the point-by-point multiplication approach has better sensitivity to detect dynamic

Figure 3c). And more interestingly, high inter-individual consistent dynamic connectivity was also found

323 between the supramarginal and default mode network (IC \# 6 and 15), which was similar to our previous

324 analysis using a different analytic approach. In contrast, the stationary connectivity showed different

325 spatial patterns than the dynamic connectivity (Figure 3b). The networks with known functional

which showed square-like structures along the diagonal.
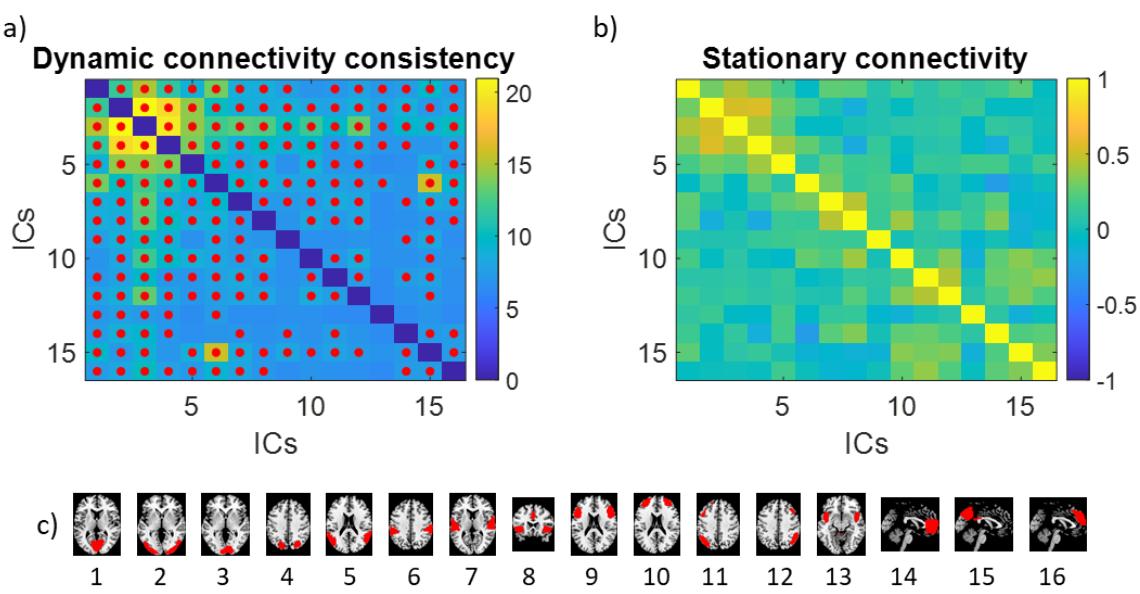

Figure 3 a) Inter-individual consistent point-by-point multiplications (dynamic connectivity) across the 
Figures $4 \mathrm{a}$ through $4 \mathrm{c}$ show the consistent point-by-point multiplications among the 16 networks

337 (ICs) for the three movie clips in the HBN-SSI dataset. Consistent with the Partly Cloudy dataset,

338 widespread consistent dynamic connectivity was observed. The networks that had more consistent

339 dynamic connectivity were higher visual networks and the auditory network (IC 7). We further directly

340 compared the consistency of dynamic connectivity among the three movies (Figure 4d through 4f).

341 Compared with the other two movie clips, the Wall-E clip showed more consistent dynamic connectivity

342 between the supramarginal network (IC 6) and many other networks, and between the posterior cingulate

343 network (IC 13) and visual related networks. Compared with the other movie clips, the clip of The

344 Matrix showed greater consistency between only three pairs of networks, among the posterior visual

345 cortex, posterior parietal network, supramarginal network, and a left frontoparietal network. And lastly,

346 compared with the other two movie clips, the A Few Good Men clip showed greater consistency in the

347 multiplication of the auditory cortex (IC 7) with other networks, and between the temporoparietal junction

348 network (IC 5) and other networks.
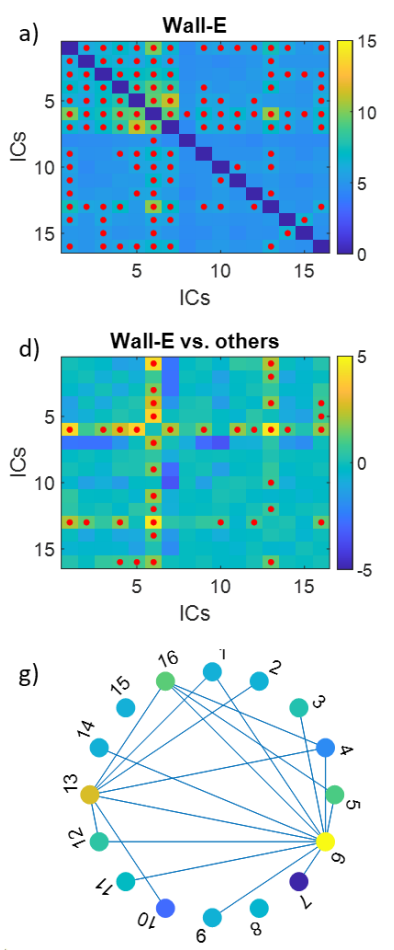
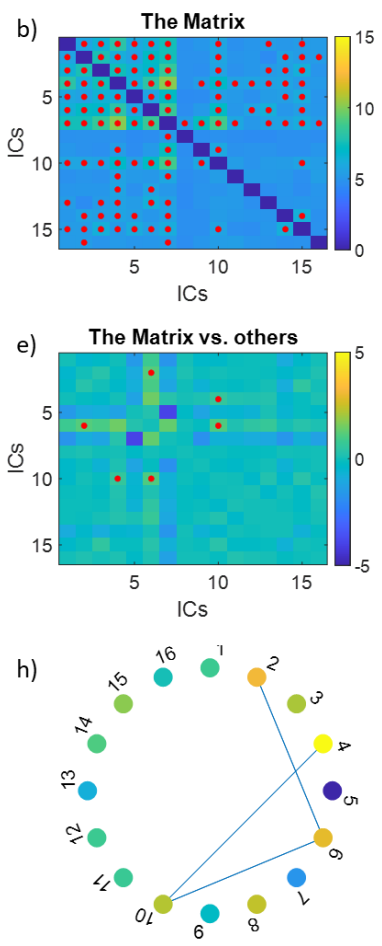
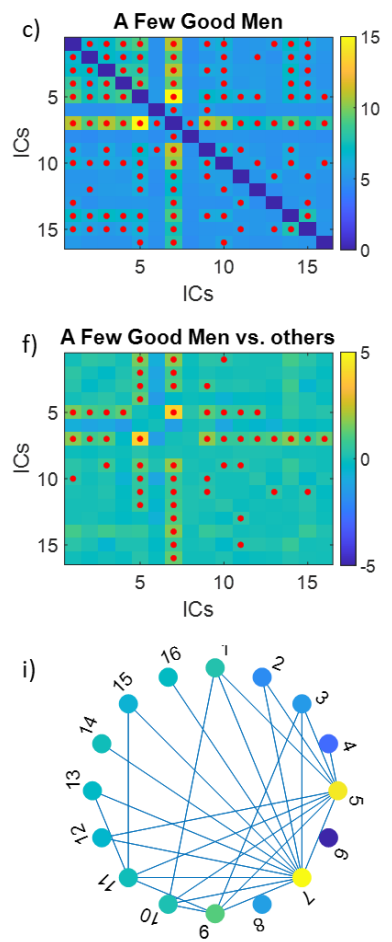

j)
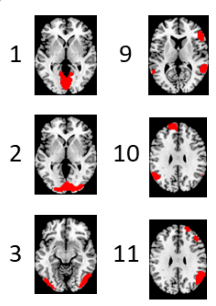

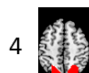
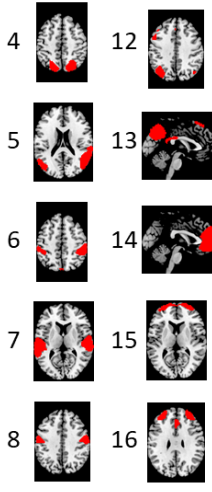
Figure 4 a) through c), consistency of dynamic connectivity across 16 networks (independent components, ICs) when watching the three movie clips in the Healthy Brain Network serial scanning

352 initiative dataset. The colors in the matrices represent the percent variance explained by the first principal

353 component of the point-by-point multiplication. d) through f), the differences in consistency of dynamic

$354 \quad$ connectivity between each movie clip and the other two clips. The red dots indicate statistical

355 significance at a false discovery rate $\mathrm{p}<0.05$ with permutation testing. The differences in consistency of

356 dynamic connectivity are also shown in graph representations in g) through i), where the node color represents the consistency differences in regional activity. j) shows the representative maps for the 16

$$
\text { network ICs. }
$$

We also examined the relationships between regional activity consistency and dynamic

370 with many consistent dynamic connectivities (Figure $4 \mathrm{~g}$ through $4 \mathrm{i}$ ).

372 surprisingly, the overall patterns in the three movies were very similar. When directly comparing the

373 differences among the three movies, no statistically significant differences were found even at the $\mathrm{p}<$

3740.05 threshold. 

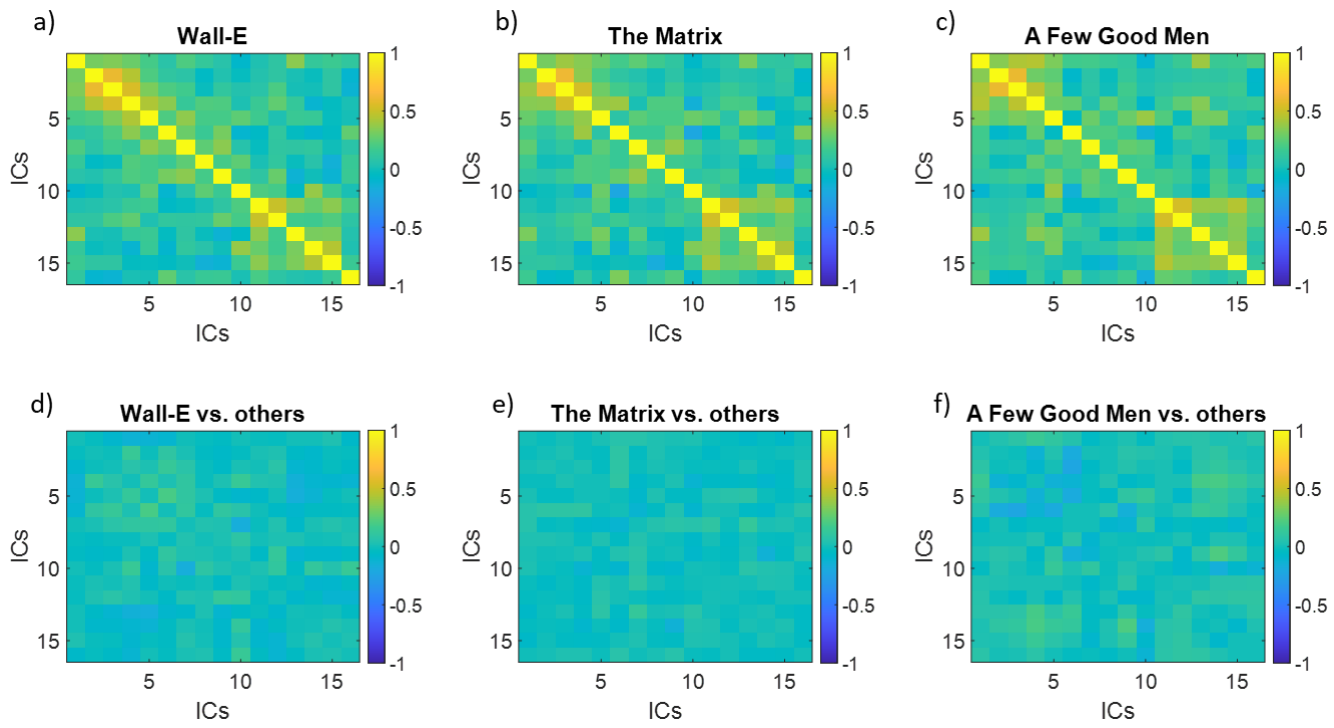

$376 \quad$ Figure 5 Top row, stationary connectivity among 16 networks (independent components, ICs) when

377 watching the three movie clips from Healthy Brain Network Serial Scanning Initiative dataset. Bottom 378 row, differences in stationary connectivity between a movie clip and the other two clips. No statistically significant difference was found even with an uncorrected threshold of $p<0.05$.

\subsection{Dynamic and stationary connectivity in 80 -IC solution}

383 We further studied the relations between dynamic and stationary connectivity in a larger spatial scale of

384 the 80-IC solution. Figure 6 shows the dynamic connectivity consistency and stationary connectivity

385 matrices for the four movie clips. The patterns are very similar to what with the 20 -IC solution. That is,

386 the stationary connectivity matrices showed modular structures, and were very similar across different

387 movie clips. In contrast, the dynamic connectivity distributions were highly skewed, with greater

388 consistency between lower-level brain regions such as the visual and auditory cortex. 

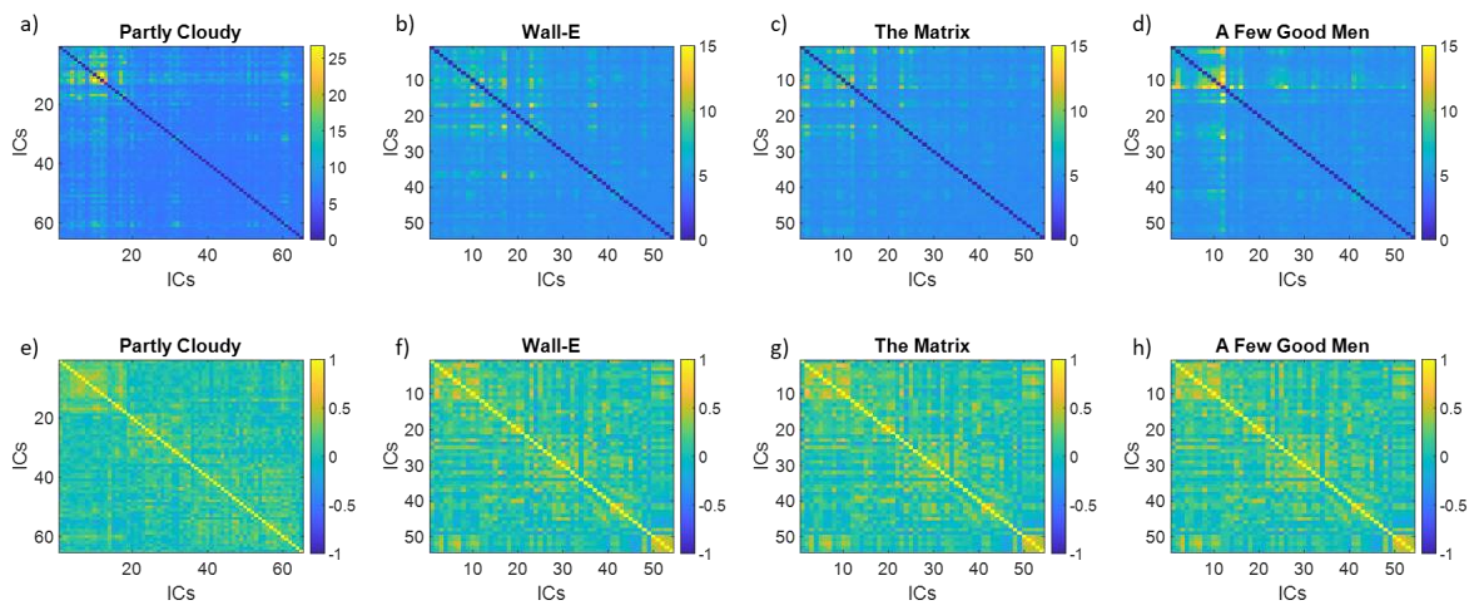

Figure 6 Dynamic connectivity consistency (top row) and mean stationary connectivity (bottom row) for the four movie clips using the 80 -independent-component solutions. Please note that the number of included independent components (ICs) are different between the Partly Cloudy dataset and the other three movie clips.

We next directly examine the correlations among the matrices. For the Partly Cloudy data, the correlation between stationary and dynamic connectivity consistency was only 0.09 (Figure 7a), although

397 it was statistically significant due to the large number of IC pairs. The relations have been confirmed by 398 the NBH-SSI dataset (Figure 7b). The stationary connectivity of the three movie clips had almost perfect 399 correlations. On the other hand, the dynamic connectivity of the three movies had moderate correlations.

400 There were very small correlations between the dynamic and stationary connectivity matrices.

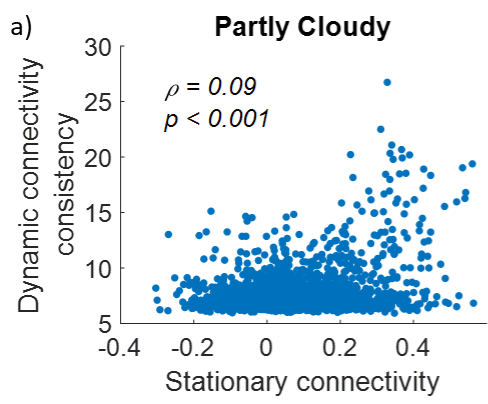

401

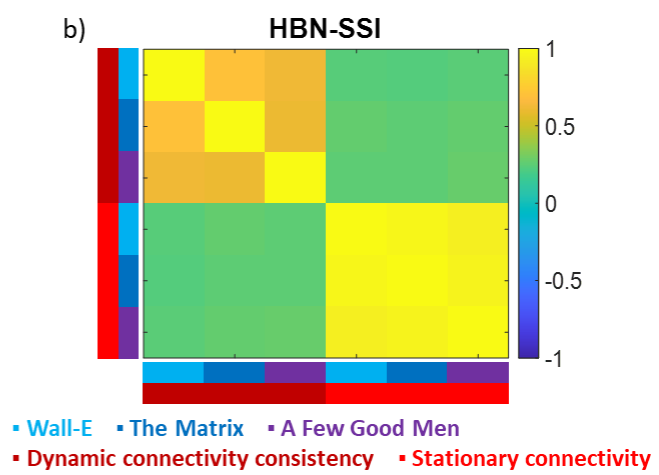


Figure 7 Correlations between stationary and dynamic connectivity for the Partly Cloudy dataset (a) and Healthy Brain Network Serial Scanning Initiative (HBN-SSI) dataset (b). The connectivity matrices were calculated based on 80-independent-component solutions. Spearman's rank correlation $(\rho)$ was used.

In order to show the spatial distributions of dynamic connectivity in the context of global

407 stationary connectivity, we calculated connectivity gradients based on the stationary connectivity of the

408 three movie clips in the HBN-SSI dataset (Top row in Figure 8). The first and second gradients

409 represented unimodal to transmodal gradient and visual to motor gradient, respectively. Next, we plotted

410 the top $10 \%$ of dynamic connectivity in each movie clip compared with the other two movie clips

411 (Bottom row in Figure 8). It can be seen that the consistent dynamic connectivity for the three movies

412 usually took place between networks from far connectivity space, connecting visual, sensorimotor, and

413 higher-order association systems. There are also notable differences among the three movie clips. For

414 example, for the movie clips A Few Good Men, the consistent dynamic connectivity connected the

415 higher-order associate areas to visual and sensorimotor regions, separately. But direct connections

416 between visual and sensorimotor regions were rare.

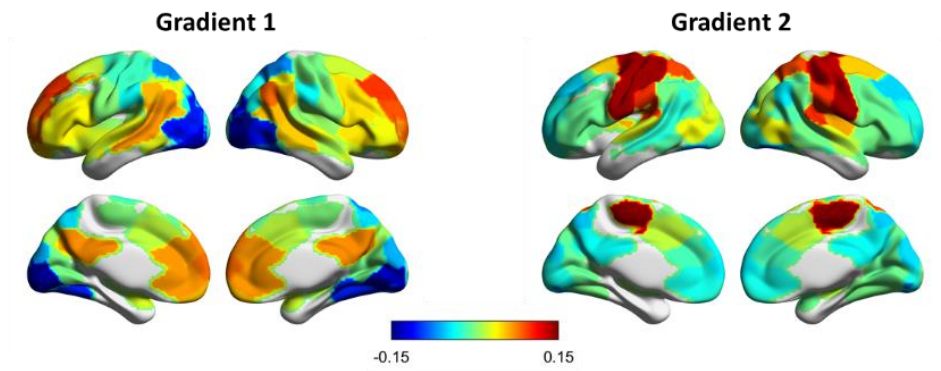

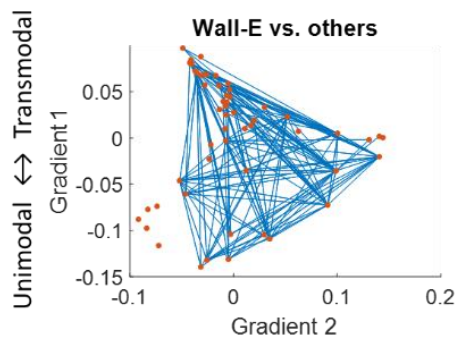

417
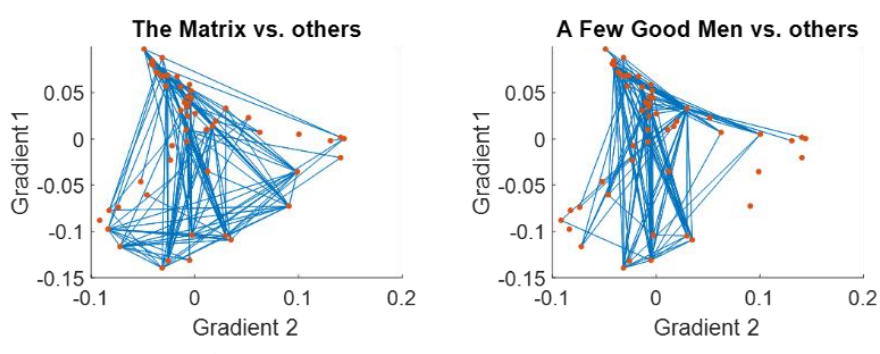
Figure 8 Top row, gradient maps of stationary connectivity in the Healthy Brain Network Serial Scanning Initiative dataset. Bottom row, top $10 \%$ consistent dynamic connectivity in each movie clip compared with the other two clips mapped to the connectivity gradient space.

\subsection{Movie clips classification}

423 Last we asked whether the dynamic or stationary connectivity pattern can enable individual-level

424 prediction of the different movie clips. For each participant from the HBN-SSI dataset, we classified one

425 of the three movie clips based on different measures. Overall, the consistency of dynamic connectivity

426 achieved the highest prediction accuracy (Accuracy $=92.6 \%)$, followed by the stationary connectivity

427 (Accuracy $=85.2 \%)$ and the consistency of regional activity $($ Accuracy $=74.1 \%)$. Compared with

428 chance level accuracy of 33.33\%, all classification accuracies were statistically significant $(p<0.001)$

429 based on permutation tests. The clip-to-clip classification results for the different features are shown in

430 Supplementary Table S1.

\section{Discussion}

433 In the current analysis, we have shown widespread dynamic connectivity that is consistent across

434 individuals when the participants watched the same movie clips. Different movie clips showed different

435 patterns of dynamic connectivity, suggesting that the moment-to-moment interactions between brain

436 regions may support the processing of context-specific information. For example, the two cartoon movie

437 clips showed similarly consistent dynamic connectivity between the posterior cingulate network and

438 supramarginal network. The action movie clip, The Matrix, showed more consistent dynamic

439 connectivity in networks related to attention. And the drama movie, A Few Good Men, showed more

440 consistent dynamic connectivity involving networks related to language processing, including bilateral

441 fronto-parietal networks and prefrontal cortex. In contrast, stationary connectivity showed very similar

442 spatial patterns in different movie clips, with few statistical differences. The dynamic connectivity 
connected brain regions that are farther in the connectivity gradient space, and can better classify different

444

movie clips than the stationary connectivity and regional activity.

We first empirically compared the statistical results of inter-individual correlations of dynamic connectivity measured by sliding window and point-by-point multiplication. Not surprisingly, the multiplication approach showed higher statistical sensitivity than the sliding window approach, as indicated by a much larger number of significant effects. It is not surprising because the point-by-point multiplication has kept the information of every time point, while the sliding-window approach can be seen as smoothed time series that could potentially filter out real signals. More specifically, the point-bypoint multiplication approach detected consistent interactions between almost every pair of network ICs. This is in line with previous studies of regional activity, which also showed statistically significant effects in almost all cortical regions (Chen et al., 2016; Di and Biswal, 2020). In contrast, the sliding window approach can only detect a small number of the dynamic connectivity among higher visual networks, and between supramarginal gyrus and posterior cingulate networks. This is probably because there is slow time-varying dynamic connectivity between these regions (e.g., Figure 1), which can be detected by the sliding window approach. This is in line with studies showing that higher-order brain regions process longer time scale information (Baldassano et al., 2017). The results confirm the limitation of the sliding window approach in studying dynamic connectivity.

Consistent dynamic connectivity is ubiquitous, but different movies clips were associated with different patterns of dynamic connectivity. The movie clips from Wall-E showed a similar dynamic connectivity pattern as another animated movie Partly Cloudy. Specifically, consistent dynamic connectivity was mainly observed in connectivity with two networks, the supramarginal and posterior cingulate networks. These regions involve higher-order social processes such as empathy and theory of mind (Richardson et al., 2018; Schurz et al., 2021). This makes sense because understanding the cartoon movies requires understanding the social interactions and intentions of the virtual characters. In contrast, the court drama clip, A Few Good Men, showed higher dynamic connectivity consistency that involved the auditory and temporoparietal junction networks. Because the court drama includes numerous 
conversations, it is not surprising that the auditory cortex dynamically interacts with other cortical areas to

470 pass auditory information to those areas. The temporoparietal junction is thought to be responsible for

471 attributing other's mental states (Koster-Hale and Saxe, 2013; Wang et al., 2021), which may also be a

472 key component in understanding the conversations in the movie clip. Of course, these brain areas also

473 involve in many other higher-order brain functions, the correlational nature of the analysis doesn't allow a

474 specific function to the observed dynamic connectivity pattens (Poldrack, 2006).

In contrast to the dynamic connectivity, no differences were identified in stationary connectivity

476 among the three movie clips. In a larger spatial scale of 54/80 ICs, we further showed that the spatial

477 distribution of stationary connectivity was highly correlated among the three movie clips, which is

478 consistent in a previous study (Vanderwal et al., 2019). The largely similar patterns of connectivity

479 during watching different movies are also in line with the observations in conventional task fMRI. When

480 regressing out task activations (Cole et al., 2014; Di and Biswal, 2019) or using continuous task design

481 (Krienen et al., 2014), the stationary connectivity or task-independent connectivity showed largely similar

482 spatial patterns with each other and with what in resting-state. Similarly, the absolute correlation patterns

483 of trial-by-trial variability of the stop and go conditions in a stop signal task also showed similar patterns

484 with each other and with a separate resting-state run (Di et al., 2020). Taken together, all the results

485 convergently suggest that there is an overall connectivity pattern that may be related to the baseline brain

486 function, but may also be related to the underlying physiology (Chen et al., 2020) or anatomical network

487 structures (Laumann and Snyder, 2021). The lack of specificity of this global connectivity pattern makes

488 it less desirable as a measure of brain connectivity in specific cognitive and mental conditions. It should

489 be noted that when using multivariate classification analysis, the spatial patterns of stationary connectivity

490 can still be used to identify different movie clips, but with less accuracy than the dynamic connectivity

491 patterns. The high classification accuracy of the dynamic connectivity suggested that dynamic

492 connectivity could potentially be useful in predictive-based analysis to reflect individual differences (Finn

493 et al., 2015). 
The spatial distributions of dynamic connectivity and stationary connectivity are largely

dissociated. On one hand, the dynamic connectivity could take place between regions within the same

496 functional systems, e.g., the visual system. This is less apparent when using the sliding-window

497 approach, probably due to that the sliding-window approach can only capture slow fluctuations of

498 dynamic connectivity. The point-by-point approach could capture the fast dynamics of the interactions.

499 It is reasonable that the lower-level sensory regions showed consistent interactions, but this may be

500 overlooked by using the conventional sliding window approach. On the other hand, the current results

501 also showed that dynamic connectivity could also take place between different functional systems, e.g.,

502 between visual areas and the default mode network. This is in line with the economic account of brain

503 network organizations, which suggests that transient communication between remote brain regions could

504 enable efficient information transmissions. When overlaying the dynamic connectivity on the

505 connectivity gradients space, it demonstrated more clearly that the dynamic connectivity took place

506 between higher association areas, such as the default mode network, and lower-level sensory or motor

507 regions.

One limitation of the current study is the sample size. The HBN serial scanning dataset has a

509 relatively small sample size $(n=9)$. However, each participant watched three different types of movie

510 clips and repeated four sessions, which enable us to directly compare connectivity among these diverse

511 movie types and ensure the robustness of the results within an individual. Although promising, a larger

512 sample size with an examination of behavioral scores is needed for future brain-behavioral association

513 studies (Finn and Bandettini, 2021), where researchers can directly examine the differences in behavioral

514 correlates between dynamic connectivity and stationary connectivity (Eichenbaum et al., 2021).

515 Secondly, the current analysis only focused on the spatial distributions of dynamic connectivity. Given

516 the ubiquitous dynamic connectivity identified in the current analysis, future studies could also examine

517 the time courses of the point-by-point multiplications, which could paint a more complete picture of the

518 dynamic connectivity. 


\section{Conclusion}

521 By analyzing the inter-individual consistency of point-by-point multiplications between brain regions, we

522 were able to identify functionally meaningful dynamic connectivity during movie watching. We found

523 that compared with the stationary connectivity, the dynamic connectivity can be more sensitive to detect

524 functional changes due to different movie contexts. The spatial distributions of dynamic connectivity and

525 stationary connectivity were largely dissociated, with dynamic connectivity more reflect long-range

526 communications. Overall, dynamic connectivity may provide more functionally related information than

527 stationary connectivity.

\section{Acknowledgment:}

This study was supported by (US) National Institutes of Health grants R15MH125332 (XD) and

R01AT009829 (BB).

\section{Conflict of interest}

The authors declare that there is no conflict of interest.

\section{References}

Allen, E.A., Damaraju, E., Plis, S.M., Erhardt, E.B., Eichele, T., Calhoun, V.D., 2014. Tracking whole-brain connectivity dynamics in the resting state. Cereb. Cortex N. Y. N 1991 24, 663-76. https://doi.org/10.1093/cercor/bhs352 Structure in Continuous Narrative Perception and Memory. Neuron 95, 709-721.e5. https://doi.org/10.1016/j.neuron.2017.06.041

Biswal, B., Yetkin, F.Z., Haughton, V.M., Hyde, J.S., 1995. Functional connectivity in the motor cortex of resting human brain using echo-planar MRI. Magn. Reson. Med. Off. J. Soc. Magn. Reson. Med. Soc. Magn. Reson. Med. 34, 537-41. https://doi.org/10.1002/mrm.1910340409

Biswal, B.B., Mennes, M., Zuo, X.-N., Gohel, S., Kelly, C., Smith, S.M., Beckmann, C.F., Adelstein, J.S., Buckner, R.L., Colcombe, S., Dogonowski, A.-M., Ernst, M., Fair, D., Hampson, M., Hoptman, M.J., Hyde, J.S., Kiviniemi, V.J., Kötter, R., Li, S.-J., Lin, C.-P., Lowe, M.J., Mackay, C., Madden, D.J., 
Madsen, K.H., Margulies, D.S., Mayberg, H.S., McMahon, K., Monk, C.S., Mostofsky, S.H., Nagel, B.J., Pekar, J.J., Peltier, S.J., Petersen, S.E., Riedl, V., Rombouts, S.A.R.B., Rypma, B., Schlaggar, B.L., Schmidt, S., Seidler, R.D., Siegle, G.J., Sorg, C., Teng, G.-J., Veijola, J., Villringer, A., Walter, M., Wang, L., Weng, X.-C., Whitfield-Gabrieli, S., Williamson, P., Windischberger, C., Zang, Y.-F., Zhang, H.-Y., Castellanos, F.X., Milham, M.P., 2010. Toward discovery science of human brain function. Proc. Natl. Acad. Sci. U. S. A. 107, 4734-9. https://doi.org/10.1073/pnas.0911855107

Bullmore, E., Sporns, O., 2012. The economy of brain network organization. Nat. Rev. Neurosci. 13, 336349. https://doi.org/10.1038/nrn3214

Calhoun, V.D., Adali, T., Pearlson, G.D., Pekar, J.J., 2001. A method for making group inferences from functional MRI data using independent component analysis. Hum. Brain Mapp. 14, 140-51.

Chen, G., Shin, Y.-W., Taylor, P.A., Glen, D.R., Reynolds, R.C., Israel, R.B., Cox, R.W., 2016. Untangling the relatedness among correlations, part I: Nonparametric approaches to inter-subject correlation analysis at the group level. Neurolmage 142, 248-259. https://doi.org/10.1016/j.neuroimage.2016.05.023

Chen, J.E., Lewis, L.D., Chang, C., Tian, Q., Fultz, N.E., Ohringer, N.A., Rosen, B.R., Polimeni, J.R., 2020. Resting-state "physiological networks." Neurolmage 213, 116707. https://doi.org/10.1016/j.neuroimage.2020.116707

Cole, M.W., Bassett, D.S., Power, J.D., Braver, T.S., Petersen, S.E., 2014. Intrinsic and task-evoked network architectures of the human brain. Neuron 83, 238-251. https://doi.org/10.1016/j.neuron.2014.05.014

Cooper, R.A., Kurkela, K.A., Davis, S.W., Ritchey, M., 2021. Mapping the organization and dynamics of the posterior medial network during movie watching. Neurolmage 236, 118075. https://doi.org/10.1016/j.neuroimage.2021.118075

Di Martino, a, Scheres, a, Margulies, D.S., Kelly, a M.C., Uddin, L.Q., Shehzad, Z., Biswal, B., Walters, J.R., Castellanos, F.X., Milham, M.P., 2008. Functional connectivity of human striatum: a resting state FMRI study. Cereb. Cortex N. Y. N 1991 18, 2735-47. https://doi.org/10.1093/cercor/bhn041

Di, X., Biswal, B.B., 2021. Principal component analysis reveals multiple consistent responses to naturalistic stimuli in children and adults 2020.05.01.073163. https://doi.org/10.1101/2020.05.01.073163

Di, X., Biswal, B.B., 2020. Intersubject consistent dynamic connectivity during natural vision revealed by functional MRI. Neurolmage 116698. https://doi.org/10.1016/j.neuroimage.2020.116698

Di, X., Biswal, B.B., 2019. Toward Task Connectomics: Examining Whole-Brain Task Modulated Connectivity in Different Task Domains. Cereb. Cortex 29, 1572-1583. https://doi.org/10.1093/cercor/bhy055

Di, X., Biswal, B.B., 2015. Characterizations of resting-state modulatory interactions in the human brain. J. Neurophysiol. 114, 2785-96. https://doi.org/10.1152/jn.00893.2014

Di, X., Zhang, Z., Biswal, B.B., 2020. Understanding psychophysiological interaction and its relations to beta series correlation. Brain Imaging Behav. https://doi.org/10.1007/s11682-020-00304-8

Eichenbaum, A., Pappas, I., Lurie, D., Cohen, J.R., D’Esposito, M., 2021. Differential contributions of static and time-varying functional connectivity to human behavior. Netw. Neurosci. 5, 145-165. https://doi.org/10.1162/netn_a_00172

Faskowitz, J., Esfahlani, F.Z., Jo, Y., Sporns, O., Betzel, R.F., 2020. Edge-centric functional network representations of human cerebral cortex reveal overlapping system-level architecture. Nat. Neurosci. 23, 1644-1654. https://doi.org/10.1038/s41593-020-00719-y

Finn, E.S., Bandettini, P.A., 2021. Movie-watching outperforms rest for functional connectivity-based prediction of behavior. Neurolmage 235, 117963. https://doi.org/10.1016/j.neuroimage.2021.117963 
Finn, E.S., Shen, X., Scheinost, D., Rosenberg, M.D., Huang, J., Chun, M.M., Papademetris, X., Constable, R.T., 2015. Functional connectome fingerprinting: identifying individuals using patterns of brain connectivity. Nat. Neurosci. 18, 1664-1671. https://doi.org/10.1038/nn.4135

Fornito, A., Harrison, B.J., Zalesky, A., Simons, J.S., 2012. Competitive and cooperative dynamics of largescale brain functional networks supporting recollection. Proc. Natl. Acad. Sci. U. S. A. 109, 12788-12793. https://doi.org/10.1073/pnas.1204185109

Freitas, L.G.A., Bolton, T.A.W., Krikler, B.E., Jochaut, D., Giraud, A.-L., Hüppi, P.S., Van De Ville, D., 2020. Time-resolved effective connectivity in task fMRI: Psychophysiological interactions of CoActivation patterns. Neurolmage 212, 116635. https://doi.org/10.1016/j.neuroimage.2020.116635

Friston, K.J., 1994. Functional and effective connectivity in neuroimaging: A synthesis. Hum. Brain Mapp. 2, 56-78. https://doi.org/10.1002/hbm.460020107

Friston, K.J., Buechel, C., Fink, G.R., Morris, J., Rolls, E., Dolan, R.J., 1997. Psychophysiological and modulatory interactions in neuroimaging. Neurolmage 6, 218-29.

Friston, K.J., Williams, S., Howard, R., Frackowiak, R.S., Turner, R., 1996. Movement-related effects in fMRI time-series. Magn. Reson. Med. Off. J. Soc. Magn. Reson. Med. Soc. Magn. Reson. Med. 35, 346-55. https://doi.org/DOI 10.1002/mrm.1910350312

Fu, Z., Chan, S.-C., Di, X., Biswal, B., Zhang, Z., 2014. Adaptive covariance estimation of non-stationary processes and its application to infer dynamic connectivity from fMRI. IEEE Trans. Biomed. Circuits Syst. 8, 228-39. https://doi.org/10.1109/TBCAS.2014.2306732

Fu, Z., Tu, Y., Di, X., Biswal, B.B., Calhoun, V.D., Zhang, Z., 2017. Associations between Functional Connectivity Dynamics and BOLD Dynamics Are Heterogeneous Across Brain Networks. Front. Hum. Neurosci. 11. https://doi.org/10.3389/fnhum.2017.00593

Hasson, U., Nir, Y., Levy, I., Fuhrmann, G., Malach, R., 2004. Intersubject synchronization of cortical activity during natural vision. Science 303, 1634-40. https://doi.org/10.1126/science.1089506

Hutchison, R.M., Womelsdorf, T., Allen, E. a., Bandettini, P. a., Calhoun, V.D., Corbetta, M., Della Penna, S., Duyn, J.H., Glover, G.H., Gonzalez-Castillo, J., Handwerker, D. a., Keilholz, S., Kiviniemi, V., Leopold, D. a., de Pasquale, F., Sporns, O., Walter, M., Chang, C., 2013. Dynamic functional connectivity: Promise, issues, and interpretations. Neurolmage 80, 360-378. https://doi.org/10.1016/j.neuroimage.2013.05.079

Kauppi, J.-P., Jääskeläinen, I.P., Sams, M., Tohka, J., 2010. Inter-subject correlation of brain hemodynamic responses during watching a movie: localization in space and frequency. Front. Neuroinformatics 4. https://doi.org/10.3389/fninf.2010.00005

Koster-Hale, J., Saxe, R., 2013. Theory of Mind: A Neural Prediction Problem. Neuron 79, 836-848. https://doi.org/10.1016/j.neuron.2013.08.020

Krienen, F.M., Yeo, B.T.T., Buckner, R.L., 2014. Reconfigurable task-dependent functional coupling modes cluster around a core functional architecture. Philos. Trans. R. Soc. Lond. B. Biol. Sci. 369, 20130526-. https://doi.org/10.1098/rstb.2013.0526

Laumann, T.O., Snyder, A.Z., 2021. Brain activity is not only for thinking. Curr. Opin. Behav. Sci., Deep Imaging - Personalized Neuroscience 40, 130-136. https://doi.org/10.1016/j.cobeha.2021.04.002

Lindquist, M.A., Xu, Y., Nebel, M.B., Caffo, B.S., 2014. Evaluating dynamic bivariate correlations in resting-state fMRI: A comparison study and a new approach. Neurolmage 101, 531-546. https://doi.org/10.1016/j.neuroimage.2014.06.052

Lurie, D.J., Kessler, D., Bassett, D.S., Betzel, R.F., Breakspear, M., Kheilholz, S., Kucyi, A., Liégeois, R., Lindquist, M.A., McIntosh, A.R., Poldrack, R.A., Shine, J.M., Thompson, W.H., Bielczyk, N.Z., Douw, L., Kraft, D., Miller, R.L., Muthuraman, M., Pasquini, L., Razi, A., Vidaurre, D., Xie, H., 
Calhoun, V.D., 2020. Questions and controversies in the study of time-varying functional connectivity in resting fMRI. Netw. Neurosci. 4, 30-69. https://doi.org/10.1162/netn_a_00116

Margulies, D.S., Ghosh, S.S., Goulas, A., Falkiewicz, M., Huntenburg, J.M., Langs, G., Bezgin, G., Eickhoff, S.B., Castellanos, F.X., Petrides, M., Jefferies, E., Smallwood, J., 2016. Situating the default-mode network along a principal gradient of macroscale cortical organization. Proc. Natl. Acad. Sci. 113, 12574-12579. https://doi.org/10.1073/pnas.1608282113

Nastase, S.A., Gazzola, V., Hasson, U., Keysers, C., 2019. Measuring shared responses across subjects using intersubject correlation. Soc. Cogn. Affect. Neurosci. 14, 667-685. https://doi.org/10.1093/scan/nsz037

O'Connor, D., Potler, N.V., Kovacs, M., Xu, T., Ai, L., Pellman, J., Vanderwal, T., Parra, L.C., Cohen, S., Ghosh, S., Escalera, J., Grant-Villegas, N., Osman, Y., Bui, A., Craddock, R.C., Milham, M.P., 2017. The Healthy Brain Network Serial Scanning Initiative: a resource for evaluating inter-individual differences and their reliabilities across scan conditions and sessions. GigaScience 6, 1-14. https://doi.org/10.1093/gigascience/giw011

Poldrack, R.A., 2006. Can cognitive processes be inferred from neuroimaging data? Trends Cogn. Sci. 10, 59-63. https://doi.org/10.1016/j.tics.2005.12.004

Richardson, H., Lisandrelli, G., Riobueno-Naylor, A., Saxe, R., 2018. Development of the social brain from age three to twelve years. Nat. Commun. 9, 1-12. https://doi.org/10.1038/s41467-018-03399-2

Salvador, R., Suckling, J., Coleman, M.R., Pickard, J.D., Menon, D., Bullmore, E., 2005. Neurophysiological architecture of functional magnetic resonance images of human brain. Cereb. Cortex N. Y. N 1991 15, 1332-42. https://doi.org/10.1093/cercor/bhi016

Schurz, M., Radua, J., Tholen, M.G., Maliske, L., Margulies, D.S., Mars, R.B., Sallet, J., Kanske, P., 2021. Toward a hierarchical model of social cognition: A neuroimaging meta-analysis and integrative review of empathy and theory of mind. Psychol. Bull. 147, 293-327. https://doi.org/10.1037/bul0000303

Simony, E., Chang, C., 2020. Analysis of stimulus-induced brain dynamics during naturalistic paradigms. Neurolmage 216, 116461. https://doi.org/10.1016/j.neuroimage.2019.116461

Thompson, W.H., Fransson, P., 2015. The mean-variance relationship reveals two possible strategies for dynamic brain connectivity analysis in fMRI. Front. Hum. Neurosci. 9. https://doi.org/10.3389/fnhum.2015.00398

Tian, Y., Margulies, D.S., Breakspear, M., Zalesky, A., 2020. Topographic organization of the human subcortex unveiled with functional connectivity gradients. Nat. Neurosci. 23, 1421-1432. https://doi.org/10.1038/s41593-020-00711-6

Vanderwal, T., Eilbott, J., Castellanos, F.X., 2019. Movies in the magnet: Naturalistic paradigms in developmental functional neuroimaging. Dev. Cogn. Neurosci. 36, 100600. https://doi.org/10.1016/j.dcn.2018.10.004

Vos de Wael, R., Benkarim, O., Paquola, C., Lariviere, S., Royer, J., Tavakol, S., Xu, T., Hong, S.-J., Langs, G., Valk, S., Misic, B., Milham, M., Margulies, D., Smallwood, J., Bernhardt, B.C., 2020. BrainSpace: a toolbox for the analysis of macroscale gradients in neuroimaging and connectomics datasets. Commun. Biol. 3, 1-10. https://doi.org/10.1038/s42003-020-0794-7

Wang, Y., Metoki, A., Xia, Y., Zang, Y., He, Y., Olson, I.R., 2021. A large-scale structural and functional connectome of social mentalizing. Neurolmage 236, 118115. https://doi.org/10.1016/j.neuroimage.2021.118115

Yeo, B.T.T., Krienen, F.M., Sepulcre, J., Sabuncu, M.R., Lashkari, D., Hollinshead, M., Roffman, J.L., Smoller, J.W., Zöllei, L., Polimeni, J.R., Fischl, B., Liu, H., Buckner, R.L., 2011. The organization of the human cerebral cortex estimated by intrinsic functional connectivity. J. Neurophysiol. 106, 1125-65. https://doi.org/10.1152/jn.00338.2011 
bioRxiv preprint doi: https://doi.org/10.1101/2021.09.14.460293; this version posted November 23, 2021. The copyright holder for this preprint (which was not certified by peer review) is the author/funder, who has granted bioRxiv a license to display the preprint in perpetuity. It is made available under aCC-BY-NC-ND 4.0 International license.

693

694

695

696

697

698

699

700
Yuan, R., Di, X., Taylor, P.A., Gohel, S., Tsai, Y.-H., Biswal, B.B., 2016. Functional topography of the thalamocortical system in human. Brain Struct. Funct. 221, 1971-1984. https://doi.org/10.1007/s00429-015-1018-7

Zhang, C., Baum, S.A., Adduru, V.R., Biswal, B.B., Michael, A.M., 2018. Test-retest reliability of dynamic functional connectivity in resting state fMRI. Neurolmage 183, 907-918. https://doi.org/10.1016/j.neuroimage.2018.08.021 